\title{
Factors influencing content credibility in Facebook's news feed
}

\author{
Inside view on the United Kingdom (UK) Post-Brexit
}

\author{
Sonia Sousa ${ }^{1,2} \mathbb{D} \cdot$ Neil Bates $^{3}$
}

Received: 7 April 2020 / Accepted: 26 January 2021 / Published online: 29 March 2021

(C) The Author(s) 2021

\begin{abstract}
This study reports an exploratory inquiry into the problematic phenomenon of fake news on Facebook, aiming at providing an inside view on how users in the United Kingdom (UK) value the credibility of news posts on Facebook in a post-Brexit era. Participants $(n=201)$ were asked to review four different Brexit-related Facebook posts that linked to news articles from UK tabloids that were published between 2016 and 2019. Two of the posts were debunked as fake news, while the other two were verified as real news. The authors of each Facebook post were different: two from UK tabloids and two from unknown individuals. Respondents were asked to identify the credibility of the news posts in Facebook's news feed. The results indicate that the author of the post significantly influences users' perceived credibility. For instance, a fake news post from an individual is perceived as the least trustworthy, while a real news post from an individual and a fake news post from a tabloid are somewhat similarly perceived. The content of a post is seen as most trustworthy when it is a real news post from a tabloid and as least credible when it is a fake news post from an individual. Finally, in two cases, credibility can predict willingness to interact with a post. The research concludes with a set of recommendations for future research.
\end{abstract}

Keywords Fake news $\cdot$ Credibility $\cdot$ Brexit $\cdot$ Human factors $\cdot$ Trust in technology

\section{Introduction}

While this democratization of mass communication is a positive development, leading to, for example, today $86 \%$ of Americans currently using the Internet and $79 \%$ having logged on to Facebook - the most popular social networks system (SNS) (Greenwood et al. 2016). It also has contributed to the rise of fake news stories and hoaxes that have been created and shared to misinform or deceive users on such platforms deliberately.

Sonia Sousa

scs@tlu.ee

Neil Bates

n.g.bates@gmail.com

1 University of Trás-os-Montes e Alto Douro (UTAD), Vila Real, Portugal

2 School of Digital technologies, Tallinn University, Narva rd 25, 10120, Tallinn, Estonia

3 Department of Multimedia and Graphic Arts, Cyprus University of Technology, 30 Arch, Kyprianos Str, 3036, Limassol, Cyprus
Huckle and White (2017) describe fake news as information without factual basis, yet presented accurately. According to Waisbord (2018), the term "fake news" fundamentally refers to fabricated information that astutely mimics news, and it is used to misinform deliberately. Levitin (2017) describes the post-truth era as a phenomenon that blurs the boundaries between fact and fiction-especially on SNS. Social network sites such as Facebook (FB) and Twitter (TW) are now referred as perpetrators of the proliferation and consumption of such false information. They are the reason for the spread and consumption of fake news, both intentionally and unintentionally nowadays. They are, also, largely responsible for fueling the spread of fake news by creating a partisan environment, as Stockdale (2017) (Li and Suh 2015) highlights. We should not, however, rush to conclude that these pronouncements about "fake news," "post-truth," and "fact-checking" movements are just a trend in contemporary news (Waisbord 2018). The term post-truth also defines a condition of public communication, for demanding a more curatorial role in the future, and to be more accountable for their social responsibilities as a critical source of information for many (Levitin 2017). 


\subsection{The problem of fake news}

The ramifications of the phenomenon became ever so apparent for the general population in the United Kingdom (UK) during and after the 2016 UK European Union (EU) membership referendum. There is increasing evidence of a coordinated effort by the Russian Government to influence British elections and the 2016 referendum through the spreading of fake news via FB groups and pages using FB's advertising network (Kopp et al. 2018). The influence of fake news has been described as unconventional warfare, the use of technology to disrupt, magnify, and distort (Althuis and Haiden 2018).

For example, before the 2016 presidential election and as reported by Isaac and Wakabayashi (2017) Isaac (2021) of The New York Times, inflammatory content from Russian operatives reached 126 million users on FB. More than 131,000 messages were published on Twitter, and over 1,000 videos uploaded to YouTube (Isaac 2021). This phenomenon is not an isolated FB problem; media outlets like Russia Today and Sputnik published 261 media articles on the EU Referendum, with an anti-EU sentiment, between 1 January 2016 and 23 June 2016 (Harris and Feldberg 2018). On TW, Russian accounts posted almost 45,000 messages about Brexit in the 48 hours before the $2016 \mathrm{EU}$ referendum (Talavera et al. 2021).

To mitigate the negative influence of the rapid dissemination of information and fake news on SNS, the UK government is currently in the process of reviewing measures designed to regulate the industry and to protect citizens from disinformation and misinformation on SNS (House of Commons 2021).

However, this problem is not taken into serious consideration in the design of information technology systems and in particular SNS. Evidence provided by a limited number of cues afforded by these social media interfaces limits the judgments on the credibility of the source, medium, and message of content stories (Keib and Wojdynski 2019).

Helping the spread of falsehoods on SNS often outperform the truth-with fake news reaching more people and being shared more quickly than accurate news. As a consequence it is now somewhat easy for fake news stories to spread and go largely undetected, and when discovered the damage has often already been done (Gulati et al. 2018; Ajenaghughrure et al. 2019).

Furthermore, in an attempt to perfect their dominance, large technology companies, such as Facebook and Google, have manipulated their algorithms to increase their reach and influence to attract an audience of millions (Illing 2021). And as indicated by McGee (2017), while popular, fact-checking among journalists is sometimes considered to be partisan.
In a society where facts seemingly no longer matter, neither does the need to fact-check. The advent of fake news can have a massive effect on the consumption of local and international news in the UK. A recent survey from Newman et al. (2019) has shown that $35 \%$ of people often or sometimes actively avoid the news. Participants indicated that the main reasons for avoiding the news were due to the negative impact on their mood, and that also they do not feel like they can do anything and they can not rely on the news to be true. The research also indicated that more than twothirds of those surveyed from the UK avoided news because of the Brexit coverage, followed by $35 \%$ indicating politics as the main reason for avoiding the news.

In an attempt for the integration of augmented measures to mitigate the negative influence of the rapid dissemination of information and fake news on SNS, the UK government is currently in the process of reviewing measures designed to regulate the industry and to protect citizens from disinformation and misinformation on SNS (House of Commons 2021), which is the same with regard to the European strategy to mitigate fake news and online disinformation and support the implementation of Ethics Guidelines for Trustworthy Artificial Intelligence (Floridi 2019; European Commission 2018).

Regardless, according to a recent study with FB users in the United States (US), it was indicated that older people are almost four times more likely to have shared fake news on FB than the younger generation (Guess et al. 2019; Huckle and White 2017). In another study, which analyzed registered US voters on TW during the 2016 presidential election, it was found that people who shared fake news were more likely to be older and more conservative (Grinberg et al. 2019). These findings indicate that there is a clear gap between the digital media literacy skills of older and younger users on SNS. Grinberg et al. (2019) also found that engagement with fake news on TW during the 2016 presidential election was too concentrated to a few sources. Based on their study, only $1 \%$ of individuals accounted for $80 \%$ of fake news shared. Moreover, their study concluded that spreaders of fake news were only a small percentage of the entire online community.

Thus, the ability to assess how much we trust a social media source's credibility is connected with online users' behavioral intentions to share and engage ( $\mathrm{Hu}$ and Shyam Sundar 2010; Sousa et al. 2011). Users rely, therefore, on the input of visual cues such as comments, likes, and shares of the post to interpret and form their perceptions of credibility (Metzger et al. 2010; Wogalter and Mayhorn 2008). When designing news feed type systems and experiences, interaction designers seek to understand the impact of those behaviors in users' engagement and continued use.

We should not, however, rush to conclude that "fake news" and "post-truth" phenomena exist only in mainstream 
journalism. Different trends and forces are at play and data journalism, and the fact-checking movement (Graves 2018) are the only conditions of the "post-truth" movement. The seeking of the truth has imposed a societal shift.

As Vosoughi et al. (2018) showed, it has reached a stage where falsehoods consistently outperform the truth-with fake news reaching more people and being shared more quickly than accurate news. With it there is proliferation on the debate on the importance of incorporating moral values into design (Manders-Huits 2011), on the risks of being unethical (Floridi 2019), and on the impact of practicing "dark patterns" to persuade or manipulate user behaviors (Hu and Shyam Sundar 2010).

With this use of dark design patterns to persuade or manipulate user experience, it proliferates conversations about ethical design (Waldman 2020) and the need for trust in technology (Oper and Sousa 2020; Fimberg and Sousa 2020), highlighting as well the effects of fake news in cognitive bias (Shu et al. 2017) in assigning trust in the information source (Trust 2018; Gulati et al. 2019).

A need arises to rethink the apparent problem that many users are not able to discern between fact or fiction (Heuer and Breiter 2018). Furthermore, people who share fake news are more likely to be older, and more conservative (Grinberg et al. 2019). With FB users in the US, older people are almost four times more likely to have shared fake news on FB than the younger generation (Guess et al. 2019).

Or the fact that interaction designers are not adequately considering the phenomenon of fake news and its impact within the experiences they build. An example is that designers often look for interaction qualities that are fun, immersive, and addictive without reflecting on the ethical ramifications of building additive and potentially misleading technologies.

This lack of trust in the credibility of the sources of the information brings the need to understand better the impact of the source (author, publication) upon perceived credibility in an attempt to support behavior patterns like sharing or engaging with social media content (Hermida et al. 2012) and also raising awareness of the role of social media platforms in ensuring information accuracy ( $\mathrm{Li}$ and Suh 2015).

In particular, this study aims to know how UK-based users' perceived credibility of real and fake news posts in FB's news feed differ. For that, a survey was designed to observe if factors like location, age, gender, education level, frequency of FB use, and intention to interact could influence content credibility in FB's news feed.

The study presented should be seen as an exploratory inquiry into the problematic phenomenon of fake news on FB rather than as a definitive conclusion.

\section{Research methodology}

As highlighted in the "Introduction" section of this paper, this research aims to understand differences in UK users' perceived credibility of real and fake news posts in FB's news feed. The study will specifically investigate the influence of author and content credibility, along with users' age, gender, education level, country (within the UK), frequency of FB use, and intention to interact with real and fake news posts.

To achieve the goal of this research, one central research question-How do UK-based users' perceived credibility of real and fake news posts on FB's news feed differ?-was defined along with six supporting hypotheses

- H1. The age of participants does not have any significant influence on their perceived credibility of FB posts.

- H2. The gender of participants does not have any significant influence on their perceived credibility of FB posts.

- H3. The education level of participants does not have any significant influence on their perceived credibility of FB posts.

- H4. The author of real and fake news posts in FB's news feed will influence users' perceived credibility of a post.

- H5. Participants' frequency of FB use does not have any significant influence on their perceived credibility of FB posts.

- H6. Participants' country of residence (within the UK) does not have any significant influence over their perceived credibility of FB posts.

- H7. The perceived credibility of a post will influence the user's interest to interact with it.

Procedure The research scope was limited to identifying differences in how UK-based users' perceive the credibility of fake, and real news posts in FB's new feed. Each participant assessed (using an online survey) the credibility of a total of four stimuli, one at a time and randomly generated, using Kang's 14-item measure for author and content credibility (Kang 2010). They indicated as well the location (the country in the UK), age, gender, education level, frequency of FB use, and intention to interact.

All survey participants were asked for consent and ensured on their anonymity as well. At the start of the survey, participants were asked to indicate whether they reside in the UK (nationality is irrelevant); those who confirmed proceeded with the survey, whereas the others were requested to exit from the survey. Similarly, data was only collect from users who are active on FB. Therefore, participants were asked if they have a FB profile. Participants who answered positively proceeded in the 
survey; this question was followed with the qualification questions, and participants were required to provide their demographic and behavioral data.

After providing demographic and behavioral data, participants entered the main part of the study, where they were presented with the stimuli. The study collected detailed information concerning their perceived credibility of real and fake news posts. In total, each participant assessed the credibility of a total of four stimuli, one at a time and randomly generated, with a 5-point Likert scale (strongly agree, agree, undecided, disagree, and strongly disagree) using Kang's (2010) 14-item measure that focuses on source and message credibility. As discussed earlier, Kang's (2010) instrument was employed to measure users perceived credibility in the context of FB's news feed. A 5point scale was used instead of the 7-point Likert scale as to prioritize simplicity and ease of use for participants in the study (Leung 2011).

After assessing each stimulus, participants were asked whether they would interact with the post, and if so, then how. This question observed how users' perceived credibility of a FB post could influence their intent to interact with the said post. Based on the data collected, this study sought only to measure and map the following variables of real and fake news examples in FB's news feed: post author credibility, post content credibility, user age, user gender, user education level, frequency of use, UK country of the user, and intention to interact with the stimuli.

Participants The data collection phase occurred in November 2019, a total of 201 participants enrolled in the study and from those 34 participants were deleted from data analysis as they failed to complete the survey in its entirety.

The majority of participants reside in England (83.8\% or 140 participants), the others in Scotland 10.2\%, Wales 4.2\%, and Northern Ireland $1.8 \%$ of the sample. Despite those representing smaller samples of the population, it reflects the actual population distribution of the UK, for example in 2018 data shows that among the 66.44 million people in UK, England represents with 84\%, Scotland 8\%, Wales 5\%, and Northern Ireland 3\% (Clark 2019).

Table 1 indicates that the majority of participants were between 30-39 years old (41.3\%) and 21-29 (22.8\%), followed by ages between 40 and 49 and ages between 50 and 59 and $60+$. The least representative age group was those aged between 18 and 20 .
When it comes to education (see Table 2), $16.2 \%$ (27 respondents) report taking undergraduate education programs but not graduating from them so far, while $23.4 \%$ (39 participants) report that they have completed their undergraduate education. There is a considerable portion of post-graduates included in the sample as well, namely 34 of them, which amounts to $20.4 \%$ of the sample. There is a bias towards the female population with $76 \%$ females and $24 \%$ males, and among the participants, $59.9 \%$ of them reported using Facebook more than once a day. The gender bias observed in the sample might be due to the criteria applied to participate in this survey.

\subsection{Survey stimuli}

For the stimuli to appear as realistic as possible, real-life examples of both real and fake news were selected. Those samples were gathered from a UK tabloid and published between 2016 and 2019. Each post was replicated as a post in FB's news feed using Photoshop, replicating an identical user interface design (UI) design of Facebook's news feed on a desktop. This intended to create a seamless experience, without the need for participants to leave the survey; image-based mockups were embedded into the survey and represented alongside Kang's (2010) instrument.

All posts are based on actual news stories that are still accessible online. Further details on each of the stimuli, the stories, and their credibility were carefully verified and outlined. Furthermore, to minimize varied bias towards the story, all of the posts featured a news story that is negative towards the EU. All four stimuli feature an actual news article from a UK tabloid that has been verified as either real or fake by one of three fact-checking initiatives: EUFACTCHECK, European Commission Representation in the UK, or Full Fact.

The author of each Facebook post is distinctive, as well as the four FB posts, which feature four different news stories: two real and two fake news stories relating to Brexit. Specifically, two posts were from a UK tabloid (Daily Mail and Daily Express) and two from unknown male individuals. The sources for each of the news stories were different, but all from UK tabloids: Daily Mail, The Sun, Express, and Mirror (see Figs. 1 and 2). Each of the posts were organic, and not sponsored and published recently.

To ensure that the stimuli presented were comparable, the following criteria was established:
Table 1 Distribution of participants across country and age, sample $(n=167)$

\begin{tabular}{lllll}
\hline Country & England (84\%) & Scotland (10\%) & Wales (4\%) & Northern Ireland (2\%) \\
Age & $18-20(5.4 \%)$ & $21-29(22.8 \%)$ & $30-39(41.3 \%)$ & \\
Age & $40-49(16.2 \%)$ & $50-59(7.2 \%)$ & $60+(7.2 \%)$ & \\
\hline
\end{tabular}


Table 2 Distribution of participants across gender and education, sample $(n=167)$

\begin{tabular}{|c|c|c|c|}
\hline Gender & Male $(24 \%)$ & Female $(76 \%)$ & \\
\hline Education & 20 with GCSE (12\%) & 25 A-Level (15\%) & 22 vocational $(13.2 \%)$ \\
\hline
\end{tabular}

- The stimuli featured four different FB posts- two real and two fake news stories relating to Brexit. Selected the UK tabloids: Daily Mail, The Sun, Express, and Mirror as a source for each of the news stories.

- The author of each post was different-two from a UK tabloid (Daily Mail and Daily Express) and two from unknown male individuals. There was one real real and one fake news story for both UK tabloid and individual author groups. Each post included different engagements (e.g., reactions, comments, and shares).

- Each of the FB posts included a link to an article from a UK tabloid. The shared link consisted of an image and headline, which was automatically generated based on the meta-data of the news article.

- Two of the posts were examples of real news stories and had been fact-checked and verified as real. In contrast, two others were examples of fake news stories that have been fact-checked and confirmed as fake.

\section{Results}

Before commencing the analysis, we first followed Tavakol and Dennick (2011) recommendation and assessed the internal consistency of the scale items using Cronbach's alpha. This is to ensure the instrument reliability to measure

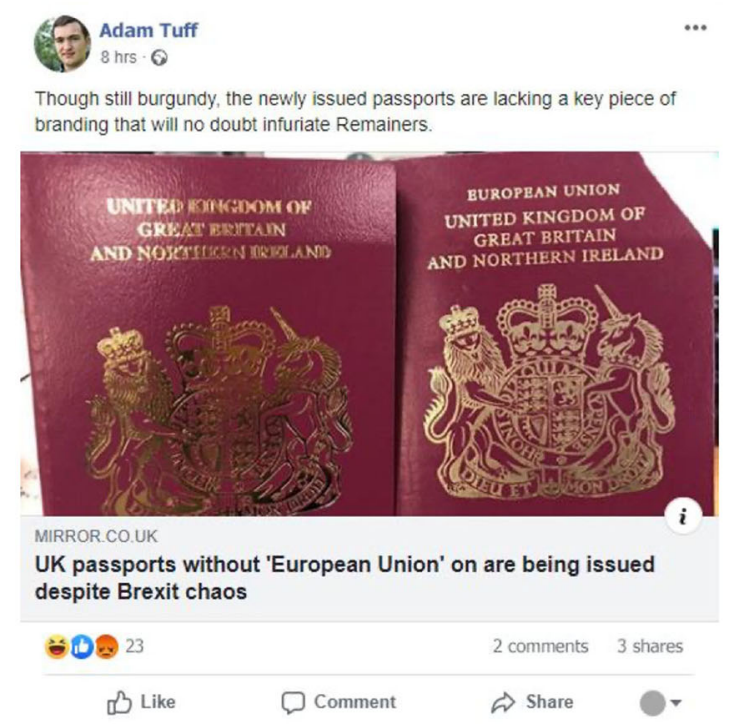

perceived credibility. The analysis proceeded, focusing on factors of authorship, whether the presented post was created by an unknown individual or by a tabloid. This analysis was followed by observing the truthfulness of the post, whether the post was real or fake. Adjunct factors to be included are country (where in the UK the participant is from), gender, age, education level, and frequency of FB use.

Scale assessment To assess the validity and reliability of the scales used and following Tavakol and Dennick (2011) recommendation that the observed Cronbach's alpha should be higher than 0.7 . We can conclude that the items of the scale demonstrated good internal consistency; see results in Table 3. Therefore, the data collected is reliable to measure UK-based users' perceived credibility of real and fake news posts on $\mathrm{FB}$.

Understanding user's perceived credibility Credibility is the most important dependent variable in the present research, and it is at the core of most hypotheses. To reiterate, the hypotheses focus on factors of authorship (whether the presented post was created by an unknown individual or by a tabloid) and the truthfulness of the post (whether the post was real or fake). Adjunct factors to be included are country (where in the UK the participant is from), gender, age, education level, and frequency of FB use.
- Design: Intercept + COUNTRY1 + FREQUENCY1 + GENDER + AGE + EDUCATION
- Within Subjects Design: factor1

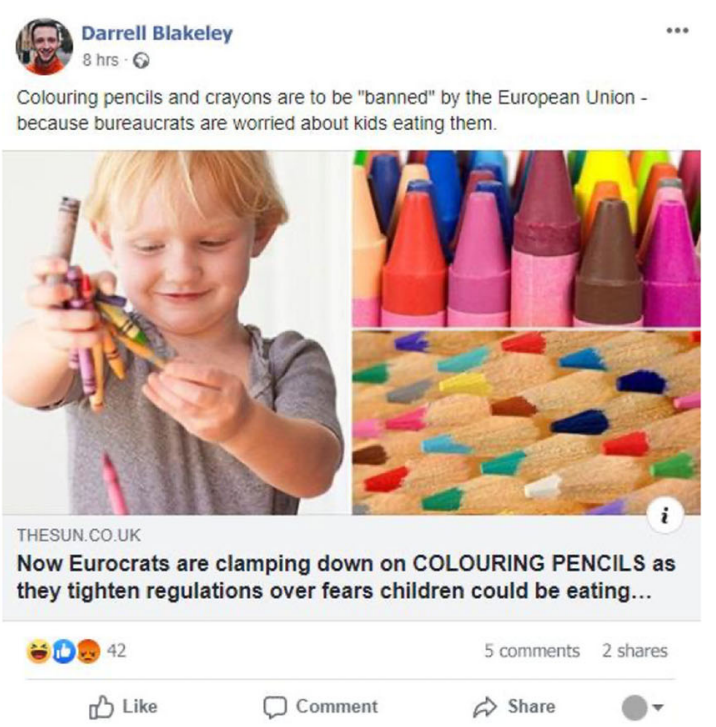

Fig. 1 Real news (left image) and fake news (right image) article shared as a Facebook post by an unknown individual 

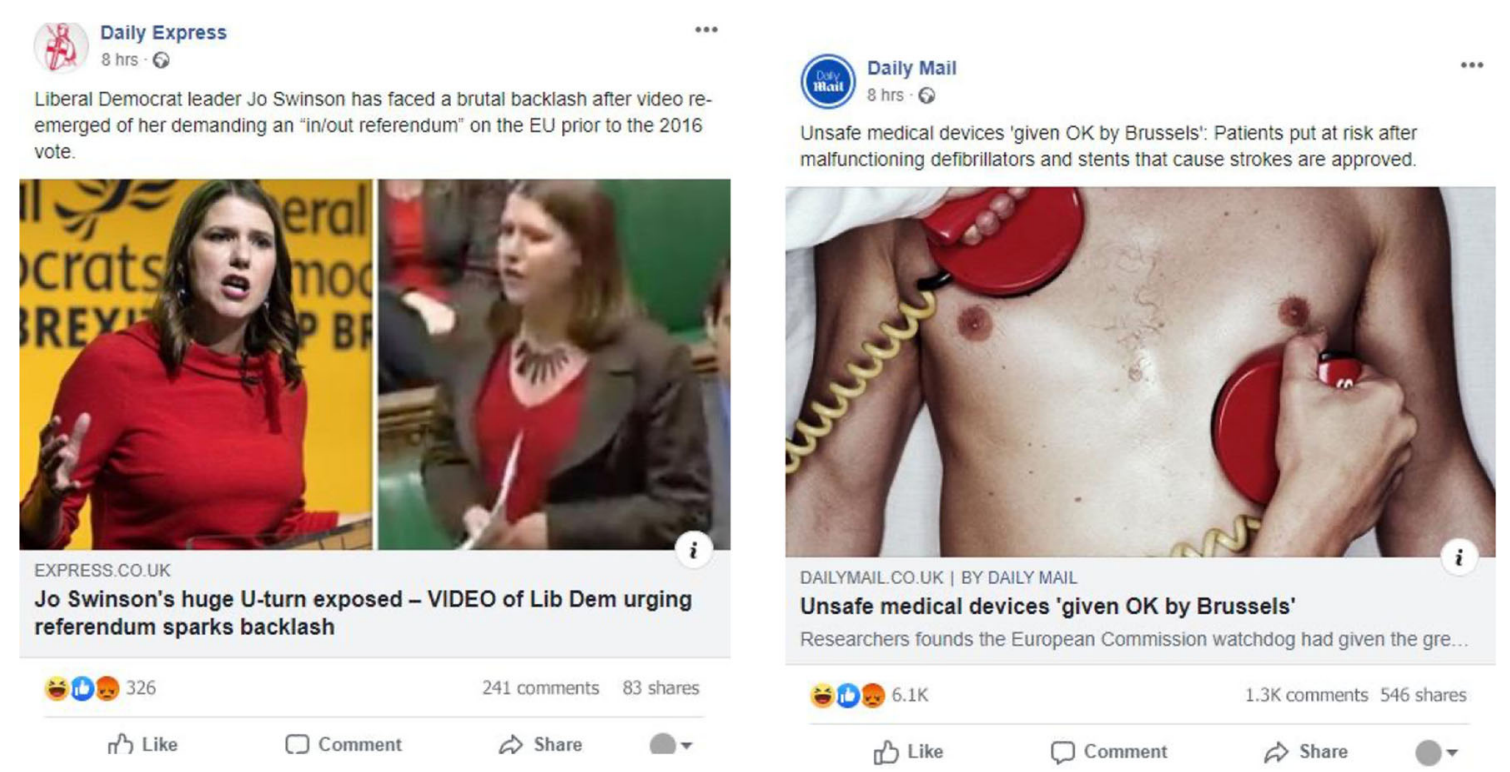

Fig. 2 Real news (left image) and fake news (right image) article shared as a Facebook post by a tabloid

In cases like this, where a researcher is presented with several independent variables, all of which are categorical and a continuous dependent variable, a general linear model is an appropriate procedure to be used Field (2013). Discovering Statistics Using IBM SPSS Statistics. Thousand Oaks, CA: SAGE.. Since all participants have responded to all stimuli, repeated measures general linear model was applied. By building a single repeated measures model where all evaluations are used as measurements and with the above listed independent variables included, the risk of type I error is greatly reduced.

Namely, it was possible to test every hypothesis separately, but that would lead to a higher risk of mentioned type I error. This error is associated with the chosen alpha level, that is, the threshold at which the $p$ value is declared statistically significant.

This is conventionally set at .05 , implying that a researcher is bringing conclusions with $95 \%$ confidence in their truthfulness. However, although a single conclusion is brought with that level of confidence, bringing several conclusions (six in order to address six hypotheses) with 95\% conclusion implies that overall level of confidence is

Table 3 Cronbach's alpha coefficients for two facets of the credibility scale proposed by Kang (2010)

\begin{tabular}{lll}
\hline Situation & $\begin{array}{l}\text { Author } \\
\text { credibility } \boldsymbol{\alpha}\end{array}$ & $\begin{array}{l}\text { Content } \\
\text { credibility } \boldsymbol{\alpha}\end{array}$ \\
\hline Real news post from individual & .716 & .853 \\
Fake news post from tabloid & .717 & .880 \\
Fake news post from individual & .711 & .861 \\
Real news post from tabloid & .826 & .912 \\
\hline
\end{tabular}

much lower, i.e., there is higher probability that some of the conclusions are wrong. By applying a single general linear model over all data simultaneously, the confidence interval remains above the $95 \%$ mark.

Judging from Table 4, it can be concluded that the condition of equality of variances has been met for every measure used. Table 4 indicates a Sig. $(p)$ value greater than .05 , thus we accept the null hypothesis and assume the variances are equal.

Before commencing the analysis, we added a new variable coding for the country dataset, due to a small number of participants in some categories. In the new dataset, participants from Wales and Northern Ireland were combined, for both items frequency of Facebook use and country. Usage that is once a week and less than once a week have been combined. The Box test of equality of covariance matrices and Mauchly test of sphericity indicate as well the following results:

Box's test of equality of covariance matrices:

- For author credibility: $F(30,998.74)=1.25$, $p=.167 ;$ and

- For content credibility: $F(30,998.74)=1.27$, $p=.150$.

Mauchly's test of sphericity:

- For author credibility: $X(5)=6.76, p=.239$; and

- for content credibility: $X(5)=4.44, p=.487$.

With both tests returning credible results, the analysis proceeded, focusing on factors of authorship whether the presented post was created by an unknown individual or by a tabloid. Judging from the results provided in Table 5 , 
Table 4 Results of Levene's test of equality of error variances

\begin{tabular}{lllll}
\hline & F & df1 & df2 & Sig \\
\hline Real news post from individual, mean & .861 & 104 & 62 & .752 \\
Fake news post from tabloid, mean & .706 & 104 & 62 & .942 \\
Fake news post from individual, mean & 1,010 & 104 & 62 & .490 \\
Real news post from tabloid, mean & .996 & 104 & 62 & .514 \\
\hline
\end{tabular}

the type of media and its truthfulness reach a statistical significance of $\mathrm{Sig}_{i} .05$. However, no significant influence was found between participants' age, gender, education, or country of residence.

H1. No significant influence between the age of participants and their perceived credibility of FB posts.

H2. No significant influence between the gender of participants and their perceived credibility of FB posts.

H3. No significant influence between the education level of participants and their perceived credibility of FB posts.

H4. Significant effects have been observed between the authors of real and fake news posts in FB's news feed and users' perceived credibility of a post.

H5. No significant influence between participants frequency of FB use and their perceived credibility of FB posts.

H6. No significant influence between participant's country of residence (within the UK) and their perceived credibility of FB posts.

In order to address hypothesis 7 stating that $(\mathrm{H} 7)$ the perceived credibility of a post will influence the user's interest to interact with it. To measure this, a binary logistic regression has been used. This method is suitable for predicting binary outcomes (would interact/would not interact) based on continuous predictors.

Findings show that fake news posts from individuals are most likely perceived as the least trustworthy. In contrast, real news posts from individuals and fake news from tabloids are somewhat similarly perceived. A sharp increase is, expectedly, observed when it comes to reading real news from tabloids.

Finally and following the hypotheses put forth earlier, it can be concluded from this sample that findings show that fake news posts from individuals are most likely perceived as the least trustworthy, while real news posts from individuals and fake news posts from tabloids are somewhat similarly perceived. A sharp increase is, expectedly, observed when it comes to reading real news from tabloids. The results of regression analyses are presented in Table 6.

An important result to take into consideration from Table 6, first, is that all four models are statistically significant. Apart from statistical significance, models show little practical significance. It is concluded that only in two cases does credibility actually predict willingness to interact with a post-for a real news post shared by an individual and a real news post shared by a tabloid.

In order to understand the contribution of author credibility, the $\operatorname{Exp}(\mathrm{B})$ values indicate how many times the chance of the outcome happening when compared to the outcome not happening for every 1-point increase in the predictor variable. The results in Table 6 illustrate that a person is 16.9 times more likely to interact with a post if its author is perceived as more credible. In brief, every point of author credibility brings an almost seventeenfold increase in the chance of users interacting with a post, if that post was real news published by an individual. If it was real news published by a tabloid, the increase is somewhat smaller and equals to 9.3. This kind of relationship, however, was not observed for fake news published by an individual and fake news published by a tabloid.

\section{Discussion}

In sum and based on the sample in this study, the following was observed:

Table 5 Results of repeated measures general linear model for credibility

\begin{tabular}{|c|c|c|c|c|c|c|c|c|c|c|}
\hline \multirow[t]{2}{*}{ Source } & \multicolumn{5}{|c|}{ Author credibility } & \multicolumn{5}{|c|}{ Content credibility } \\
\hline & $\mathrm{F}$ & df1 & $\mathrm{df} 2$ & $\mathrm{p}$ & $\eta^{2}$ & $\mathrm{~F}$ & df1 & df 2 & $\mathrm{p}$ & $\eta^{2}$ \\
\hline Repeated & 3.85 & 3 & 450 & $.010 *$ & .025 & 5.88 & 3 & 450 & $.001 *$ & .038 \\
\hline Country*Repeated & 1.66 & 6 & 450 & .131 & .022 & .44 & 6 & 450 & .853 & .006 \\
\hline Frequency*Repeated & .79 & 9 & 450 & .626 & .016 & .20 & 9 & 450 & .995 & .004 \\
\hline Gender*Repeated & .58 & 3 & 450 & .632 & .004 & .12 & 3 & 450 & .951 & .001 \\
\hline Age*Repeated & .81 & 15 & 450 & .667 & .026 & 1.53 & 15 & 450 & .090 & .049 \\
\hline Education*Repeated & .98 & 15 & 450 & .476 & .032 & .968 & 15 & 450 & .488 & .031 \\
\hline
\end{tabular}


Table 6 Results of repeated measures general linear model for credibility

\begin{tabular}{|c|c|c|c|c|c|c|}
\hline & $\mathrm{B}$ & S.E. & Wald & df & $p$ & $\exp (B)$ \\
\hline \multicolumn{7}{|c|}{ Real news post from individuals $\left(\mathrm{a}^{\mathrm{a}}\right)$} \\
\hline Author credibility & 2.828 & 1.136 & 6.197 & 1 & $.013 *$ & 16.913 \\
\hline Content credibility & -.692 & .924 & .560 & 1 & .454 & .501 \\
\hline Constant & -8.307 & 2.322 & 12.801 & 1 & .000 & .000 \\
\hline \multicolumn{7}{|c|}{ Fake news post from tabloid $\left(\mathrm{b}^{\mathrm{b}}\right)$} \\
\hline Author credibility & .875 & .888 & .971 & 1 & .324 & 2.399 \\
\hline Content credibility & .409 & .892 & .211 & 1 & 646 & 1.506 \\
\hline Constant & -6.241 & 1.592 & 15.368 & 1 & .000 & .002 \\
\hline \multicolumn{7}{|c|}{ Fake news post from individual $\left(\mathrm{c}^{\mathrm{c}}\right)$} \\
\hline Author credibility & 1.015 & .744 & 1.859 & 1 & .173 & 2.759 \\
\hline Content credibility & .551 & .727 & .574 & 1 & .449 & 1.734 \\
\hline Constant & -5.919 & 1.694 & 12.205 & 1 & .000 & .003 \\
\hline \multicolumn{7}{|c|}{ Real news post from tabloid $\left(\mathrm{d}^{\mathrm{d}}\right)$} \\
\hline Author credibility & 2.232 & .832 & 7.192 & 1 & $.007 *$ & 9.315 \\
\hline Content credibility & -.725 & .915 & .628 & 1 & .428 & .484 \\
\hline Constant & -6.627 & 1.662 & 15.905 & 1 & .000 & .001 \\
\hline
\end{tabular}

${ }^{a}$ Model fit: $2(2)=10.88, \mathrm{p}=.004$, Nagelkerke R2 $=.150$

bodel fit: $2(2)=6.10, \mathrm{p}=.047$, Nagelkerke R2 $=.093$

${ }^{\mathrm{c}}$ Model fit: $2(2)=7.70, \mathrm{p}=.021$, Nagelkerke R2 $=.087$

${ }^{\mathrm{d}}$ Model fit: $2(2)=14.81, \mathrm{p}=.001$, Nagelkerke R2 $=.176$
User demographics No influence observed. The age, gender, and education level of participants do not have any significant influence on their perceived credibility of FB posts. The same for frequency of FB use and country (within the UK).

Post author credibility Influence observed. The study has made it evident that the author of a FB post significantly influences users' perceived credibility of posts, a fake news post from an individual is perceived as the least trustworthy, while a real news post from an individual and a fake news post from a tabloid are somewhat similarly perceived.

Post content credibility Influence observed. The content of a post is seen as most trustworthy when it is a real news post from a tabloid and as least credible when it is a fake news post from an individual.

Intention to interact Influence observed. In two cases, it was found that perceived credibility can predict willingness to interact with a post (for a real news post shared by an individual and real news post shared by a tabloid). Participants were 16.9 times more likely to interact with a real news post from an individual if its author was perceived as more credible.

Important practical conclusions can be drawn from these results about the predictability of interaction. Perhaps one of the largest limitations in the sample is not the total sample size, but rather the positive response rate to participant in interacting with a post. Ten percent of respondents claimed that they would interact with at least one of the posts and $1.8 \%$ stating that they would interact with all four posts. As the model predicts that no one will interact (a system-default first step), it will still score around $90 \%$ of correct predictions, a mark that is hard to improve upon. By developing different FB posts, future researchers could focus stimuli to provoke reactions to them more easily. Only when a considerable proportion of the sample claims that they intend to interact with a given post can a sound statistical estimation be made.

\section{Conclusion}

As highlighted throughout this paper, the outcome of this research aims to help tackle the problem of fake news on $\mathrm{FB}$, especially providing an inside view on how UK-based users value the credibility of news in a post-Brexit era.

The study in this research has made it evident that the author of a post significantly influences users' perceived credibility. Interestingly, the credibility of real news from an unknown individual was seen to be perceived similarly as fake news from tabloids. 
This highlights the trust placed in mainstream news media and their duty to ensure what they report to their readers is factual, even on SNS. With regard to content credibility of posts, unsurprisingly, the study found that content is seen as most trustworthy when it is a real news post from a tabloid and as least credible when it is a fake news post from an individual.

The study has shown that by measuring users' perceived credibility of FB posts, in some cases, it is possible to predict willingness to interact with a post. For example, it was found that users are 16.9 times more likely to interact with a real news post from an individual if the author is perceived as more credible.

This is significantly higher than the likelihood of interacting with a real news post from a tabloid. Participants in the study were 9.3 times more likely to interact with a real news post from a tabloid if the author is perceived as more credible.

As already mentioned, the research has shown the significance of post authors on users' perceived credibility. It appears that, generally, participants perceive news posts from tabloids as more credible; however, when they do perceive posts to be credible from individuals, it appears to have more weight on users ' intention to interact.

Based on the significant findings in this research, the following recommendations are put forward:

Highlighting the reputation of authors Designers and engineers of news feed type systems and experiences should investigate the possibilities associated with verifying and highlighting the reputation and credibility of the content being shared and also the profiles of the organizations or individuals who are sharing the content. This could potentially be achieved through feedback from the community or through machine learning by analyzing the previous activity of a profile.

Halting interaction with disputed content Concerning content credibility irrespective of the author, designers, and engineers should investigate ways in which posts with content considered to be of low credibility could be stripped of certain features. This could work by limiting interaction with disputed content by removing engagement features in order to curb the spread of disputed information. This could be a temporary measure until the content has been actually verified by artificial intelligence or a human moderator. Also, as already being tested more broadly on FB and Instagram, there is possible potential in hiding engagement indicators such as likes and comments only on posts that are currently flagged as fake news.

A wider range of authors and stimuli While it is evident that posts from tabloids are generally seen as more credible than posts from unknown individuals, researchers could explore perceived credibility in relation to known individuals such as friends, families, colleagues, political figures, or celebrities. Future researchers should also consider using a wider range of stimuli than used in this study in order to investigate other contributing factors such as the timing of the post, the number of engagements, and varying sources.

New measurement instrument Finally, future researchers should focus on developing methods to understand whether means of post presentation affect its perceived credibility, in the hope of building a more comprehensive credibility assessment than the one proposed by Kang (2010). Additionally, future research in this area could use more than one post per situation in order to provide a more reliable estimate for a condition.

\section{Declarations}

I declare that, apart from work whose authors are clearly acknowledged, this manuscript is a product of the author's original work, and it has not been published before, neither is it currently being considered for publication elsewhere for any other comparable academic degree. A preliminary report of the work reported was published in the International Conference on Applied Human Factors and Ergonomics AHFE 2020: Advances in Artificial Intelligence, Software and Systems Engineering, pp. 174-180. Bates, N. and Sousa, S. C. (2021). Investigating users' perceived credibility of real and fake news posts in facebook's news feed: Uk case study. In Ahram, T., editor, Advances in Artificial Intelligence, Software and Systems Engineering, pages 174-180, Cham. Springer International Publishing.

Conflict of interest The authors have no mpeting interests.

Open Access This article is licensed under a Creative Commons Attribution 4.0 International License, which permits use, sharing, adaptation, distribution and reproduction in any medium or format, as long as you give appropriate credit to the original author(s) and the source, provide a link to the Creative Commons licence, and indicate if changes were made. The images or other third party material in this article are included in the article's Creative Commons licence, unless indicated otherwise in a credit line to the material. If material is not included in the article's Creative Commons licence and your intended use is not permitted by statutory regulation or exceeds the permitted use, you will need to obtain permission directly from the copyright holder. To view a copy of this licence, visit http:// creativecommonshorg/licenses/by/4.0/.

\section{References}

Greenwood S, Perrin A, Duggan M (2016) Social media update 2016. Pew Research Center 11(2)

Huckle S, White M (2017) Fake news: a technological approach to proving the origins of content, using blockchains. Big data 5(4):356

Waisbord S (2018) Truth is what happens to news: on journalism, fake news, and post-truth. Journal Stud 19(13):1866 
Li R, Suh A (2015) Factors influencing information credibility on social media platforms: evidence from Facebook pages. Procedia Comput Sci 72:314

Levitin DJ (2017) Weaponized lies: how to think critically in the post-truth era

Kopp C, Korb KB, Mills B (2018) Written evidence to the inquiry on disinformation and 'fake news', house of commons digital, culture, media and sport committee

Althuis J, Haiden L (2018) Fake news: a roadmap

Isaac M (2021). W. D. Russian influence reached 126 Million through Facebook alone. https://www.nytimes.com/2017/10/ 30/technology/facebook-google-russia.html[Accessed:January, 2020]

Harris M, Feldberg J (2018). 89up releases report on russian influence in the eu referendum. Report, 89up. http://89up.org/ russia-report[Accessed:January, 2020]

Talavera O, Pham T, Gorodnichenko Y (2021) Research suggests bots generated social media stories during EU Referendum. https://www.swansea.ac.uk/press-office/latest-research/research suggestsbotsgeneratedsocialmediastoriesduringeureferendum. php[Accessed: January,2020]

House of Commons (2021) Digital, culture, media and sport committee: disinformation and ?fake news?: Interim Report. https:// publications.parliament.uk/pa/cm201719/cmselect/cmcumeds/ 363/363.pdf[Accessed:January,2020]

Keib K, Wojdynski B (2019) Staying alive: TV news Facebook posts, perceived credibility, and engagement intent. Electronic News 13(1):3

Gulati S, Sousa S, Lamas D (2018) Modelling trust in human-like technologies. In: Proceedings of the 9th Indian conference on human computer interaction, pp 1-10

Ajenaghughrure IB, Sousa S, Kosunen IJ, Lamas D (2019) Predictive model to assess user trust: a psycho-physiological approach. In: Proceedings of the 10th Indian conference on human-computer interaction, pp 1-10

Illing S (2021) The EU just fined google, but tech companies became too big long ago. https://www.vox.com/technology/2017/9/22/ 16330008/eu-fines-google-amazon-monopoly-antitrustregulation[Accessed:January,2020]

Newman N, Fletcher R, Kalogeropoulos A, Nielsen R (2019) Reuters institute digital news report 2019

Floridi L (2019) Translating principles into practices of digital ethics: five risks of being unethical. Philos Technol 32(2):185

European Commission (2018) Synopsis report of the public consultation on fake news and online disinformation. https://ec. europa.eu/digital-single-market/en/news/synopsis-report-publicconsultation-fake-news-and-online-disinformation[Accessed: January,2020]

Guess A, Nagler J, Tucker J (2019) Less than you think: prevalence and predictors of fake news dissemination on Facebook. Sci Adv 5(1):eaau 4586

Grinberg N, Joseph K, Friedland L, Swire-Thompson B, Lazer D (2019) Fake news on Twitter during the 2016 US presidential election. Science 363(6425):374

$\mathrm{Hu}$ Y, Shyam Sundar S (2010) Effects of online health sources on credibility and behavioral intentions. Commun Res 37(1):105

Sousa S, Lamas D, Dias P (2011) The interrelation between communities, trust and their online social patterns. In: IEEE Ninth international conference on dependable, autonomic and secure computing (IEEE), vol 2011, pp 980-986

Metzger MJ, Flanagin AJ, Medders RB (2010) Social and heuristic approaches to credibility evaluation online. J Commun 60(3):413

Wogalter MS, Mayhorn CB (2008) Trusting the Internet: cues affecting perceived credibility. Int $\mathbf{J}$ Technol Human Interact (IJTHI) 4(1):75

Graves D (2018) Understanding the promise and limits of automated fact-checking

Vosoughi S, Roy D, Aral S (2018) The spread of true and false news online. Science 359(6380): 1146

Manders-Huits N (2011) What values in design? The challenge of incorporating moral values into design. Sci Eng Ethics 17(2):271

Floridi L (2019) Establishing the rules for building trustworthy AI. Nat Mach Intell 1(6):261

Waldman AE (2020) Cognitive biases, dark patterns, and the ?privacy paradox? Curr Opin Psychol 31:105

Oper T, Sousa S (2020) User attitudes towards Facebook: perception and reassurance of trust (Estonian Case Study). In: International conference on human-computer interaction. Springer, pp 224 230

Fimberg K, Sousa S (2020) The impact of website design on users? trust perceptions. In: International conference on applied human factors and ergonomics. Springer, pp 267-274

Shu K, Sliva A, Wang S, Tang J, Liu H (2017) Fake news detection on social media: a data mining perspective. ACM SIGKDD Explor Newslett 19(1):22

Trust NL (2018) Fake news and critical literacy: the final report of the commission on fake news and the teaching of critical literacy in schools. Report, National Literacy Trust. https://literacytrust.org.uk/research-services/research-reports/ fake-news-and-critical-literacy-final-report/

Gulati S, Sousa S, Lamas D (2019) Design, development and evaluation of a human-computer trust scale. Behav Inf Technol 38(10):1004. https://doi.org/10.1080/0144929X.2019.1656779

Heuer H, Breiter A (2018) Trust in news on social media. In: Proceedings of the 10th Nordic conference on human-computer interaction, pp 137-147

Hermida A, Fletcher F, Korell D, Logan D (2012) Share, like, recommend: decoding the social media news consumer. J Stud $13(5-6): 815$

Kang M (2010) Measuring social media credibility: a study on a Measure of Blog Credibility. Institute for Public Relations. pp 59-68

Leung SO (2011) A comparison of psychometric properties and normality in 4-, 5-, 6-, and 11-point Likert scales. J Soc Serv Res 37(4):412

Clark D (2019) Population of the UK, by country 2018. https://www. statista.com/statistics/294729/population-united-kingdom-uk-bycountry/[Accessed:January,2020]

Tavakol M, Dennick R (2011) Making sense of Cronbach's alpha. Int J Med Ed 2:53

Field A (2013) Discovering Statistics Using IBM SPSS Statistics. Thousand Oaks, CA:SAGE

Publisher's note Springer Nature remains neutral with regard to jurisdictional claims in published maps and institutional affiliations. 